resistance in certain clones of Solanum andigenum (a tuber-forming tetraploid close to $S$. tuberosum) and in the species $S$. vernei (a wild diploid). Repeated back-crossing of the andigenum $\times$ tuberosum hybrid had led to very promising results, but these hybrids produced an active 'hatching factor' and the resultant hatched larvæ readily penetrated the roots, where they stimulated the formation of giant cells just as in normal potatoes. Although only males completed their development in hybrid roots, so that an eelworm population would be rapidly reduced by a succession of these crops, nevertheless this mass invasion of the roots led to a reduced yield of tubers. Worse, there was now evidence of an eelworm strain to which these hybrids were not resistant. Thus, the (at first) less-promising diploid, $S$. vernei, might in the long run prove the more reliable source of resistance.

In the third paper, R. S. Pitcher and J. E. Crosse (East Malling) discussed a very different eelworm, the ectoparasitic leaf-eelworm, A phelenchoides ritzemabosi, which causes trouble in strawberries and other plants. Some years ago, these two workers showed that this eelworm could, in conjunction with Corynebacterium fascians, cause a curious condition called cauliflower disease. The interest of the phenomenon lay in the fact that both pathogens were required in order to get the very typical cauliflower symptoms in the strawberry plant. On the present occasion, Dr. Pitcher went through the subsequent experimental work which was necessary to prove the duplex origins of the disease. It was not enough to have the four categories: with and without eelworm, with and without bacterium (giving either, both, and neither), since in fact wild strains of the bacterium kept vitiating results. In the end, strawberry seedlings had to be grown from sterilized seed by sterile culture methods and eelworms had to be rendered sterile also. It was then possible to show that each pathogen could cause a characteristic symptom, but only together would they produce cauliflower disease.

The ensuing discussion had the effect of underlining the patchwork nature of present knowledge in this field. The plant-parasitic nematodes are not only of great academic interest, readily lending themselves to experimental methods of investigation; they are also of considerable economic importance.

B. G. Peters

\section{A NEW MICROWAVE AMPLIFIER}

DROF. C. H. TOWNES and his associates at Columbia University described in 1955 (Phys. Rev., 99, 1264) a new type of microwave amplifier for which they coined the name 'maser'-microwave amplification of stimulated emission of radiation. The amplification is produced by stimulated emission of radiation from systems in which a higher energy level is more densely populated than a lower one. Townes first demonstrated such an amplifier using molecular beams in gases. Attempts to apply the principle to solids have until very recently met with only partial success.

Last October, N. Bloembergen (Phys. Rev., 104, 324 ; 1956) directed attention to the possible use of power saturation of one transition in a multiple energy level system to obtain a change of sign of the population between another pair of levels. Now paramagnetic substances are characterized by an unpaired spinning electron and an inversion of the spin levels can be obtained. The particular group known as 'ionically bound paramagnetic salts' are very suitable, and Bloembergen showed theoretically that crystals of nickel fluosilicate or gadolinium ethyl sulphate at liquid helium temperatures would be satisfactory to use for a low noise microwave amplifier, or frequency converter, that is, for a solid state 'maser'.

The first successful operation of a 'maser' of this type, using a crystal of lanthanum ethyl sulphate containing about 0.5 per cent $\mathrm{Gd}+++$ and 0.2 per cent $\mathrm{Ce}^{+++}$in contact with liquid helium at $1.2^{\circ} \mathrm{K}$., was demonstrated by H. E. D. Scovil, G. Feher and H. Seidel at the Bell Telephone Laboratories, Murray Hill, New Jersey, on November 27, and a brief description has since been published (Phys. Rev., 105,762 ; 1957). The magnetically dilute para. magnetic salt crystal must have at least three energy levels the transitions of which fall within the microwave frequency range, and the basic operation can be explained by considering these three different energy levels for the unpaired spinning electrons in the crystal lattice for a large applied magnetic field. Normal electron spin states are such that the number occupying state 1 (the lowest energy state) exceeds the number in state 2, which in turn exceeds the number in state 3 (the highest energy state). By irradiating the crystal with sufficient microwave power of the proper frequency, transitions from state 1 to state 3 take place until the populations of these two states are essentially equal (power saturation). Under these conditions, the population of state 2 can be made greater than that of state 1 , and if another, smaller, microwave signal of frequency corresponding to the energy difference between the states 2 and 1 is applied, stimulated transitions will occur, giving rise to radiation with consequent power gain.

In the experimental 'maser' a saturating magnetic field at $17.52 \mathrm{kMc} / \mathrm{s}$. was used to induce transitions between the $-5 / 2$ (the lowest) and the $-1 / 2$ (the highest) energy states in the ground state of the gadolinium ion, and a signal at $9.06 \mathrm{kMc}$./s. was applied between the $-5 / 2$ and the $-3 / 2$ states. The crystal was mounted in a wave-guide cavity which was simultaneously resonant to the two frequencies, and occupied about 8 por cent of the cavity volume, being located at the point of maximum field intensity. Low-temperature operation is necessary in order that power saturation of the crystal takes place for a reasonable amount of energizing oscillator power and also to increase the population difference between the energy levels. For the required separation of the electron spin energy levels a d.c. magnetic field of 2,850 oersteds was applied at an angle of $17^{\circ}$ to the normal to the crystal, but this magnetic field may be altered to change the electron spin energies and thus tume the frequency of the stimulated radiation. The graph of output power at $9 \mathrm{kMc} / \mathrm{s}$. against input power at $17.5 \mathrm{kMc} / \mathrm{s}$. shows an output of 15 microwatts for an input of 200 milliwatts, but the particular merit of the solid state 'maser' is its very low inherent noise (corresponding to thermal noise of $5-10^{\circ} \mathrm{K}$.) compared with ordinary electronic oscillators or amplifiers which depend on the motion of charged particles at high temporatures.

It should be possible with this device to amplify extremely weak radio signals-signals which may be several hundred times weaker than those at present usable-and with proper choice of solid-state materials and operating conditions, the solid-state 'maser' should be a valuable pre-amplifier in a wide variety of microwave applications. $\quad$ S. WEINTroub 